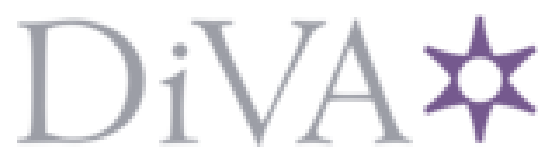

http://www.diva-portal.org

\title{
Postprint
}

This is the accepted version of a paper presented at Academic MindTrek 2015, 22-24 September 2015, Tampere, Finland.

Citation for the original published paper:

Nyström, T., Mustaquim, M. (2015)

Managing Framing Effects in Persuasive Design for Sustainability.

In: Turunen, M (ed.), Proceedings of the 19th International Academic MindTrek Conference

(AcademicMindTrek '15) (pp. 122-129). New York: Association for Computing Machinery

(ACM)

http://dx.doi.org/10.1145/2818187.2818277

N.B. When citing this work, cite the original published paper.

Permanent link to this version:

http://urn.kb.se/resolve?urn=urn:nbn:se:uu:diva-261097 


\section{Managing Framing Effects in Persuasive Design for Sustainability}

\author{
Tobias Nyström \\ Uppsala University \\ Uppsala, Sweden \\ tobias.nystrom@im.uu.se
}

\author{
Moyen M. Mustaquim \\ Uppsala University \\ Uppsala, Sweden \\ moyen.mustaquim@im.uu.se
}

\begin{abstract}
For decades, the framing effect has been a popular research topic in social psychology. A persuasive system could act in shaping the user behaviour towards sustainability, and thereby play a key role in social change. In this respect, understanding different framing effects in persuasive design is crucial, since individual framing and cognition on sustainability plays a major role in the success of persuasion. For dealing with a complex issue, such as achieving sustainability through behavioural change, persuasive design needs to reflect on the potentials of framing effects. In this paper, we have proposed a model of framing effects from which the principles of managing different framing effects of persuasive sustainability design were formulated. The principles were then shown in relations with different dimensions of sustainability and were also used to generate a design space for persuasive sustainability. The proposed model and principles are significant for realizing the effectiveness of different framing effects for the success of a persuasive sustainability.
\end{abstract}

\section{Categories and Subject Descriptors}

H.5.m. [Information interfaces and presentation (e.g., HCI)]: Miscellaneous.

\section{General Terms}

Design, Theory.

\section{Keywords}

Sustainability; System Design; Sustainable System; Framing Effect; Framing Effect Management.

\section{INTRODUCTION}

Information system design for sustainability is important, considering the current effort put forward by our society to

"Copyright is held by the owner/author(s), 2015. Publication rights licensed to ACM. This is the author's version of the work. It is posted here by permission of ACM for your personal use. Not for redistribution. The definitive version was published in AcademicMindTrek '15, September 22 - 24, 2015, Tampere, Finland http://doi.acm.org/10.1145/2818187.2818277 ". find solutions that contribute to a better and more sustainable future development. Persuasive design for sustainability is a popular topic of interest in various multi-disciplinary research fields like information system (IS) and human-computer interaction (HCI) that blends psychology, computer science, business, design, and sociology. Fogg et al. [11] states that "persuasion is a non-coercive attempt to change attitudes or behaviours" and following this Oinas-Kukkonen and Harjumaa [28] defines persuasive systems as "a computerized software or IS designed to reinforce, change or shape attitudes or behaviours or both without using coercion or deception." A lot of persuasive technology research has been aimed at health issues, for example: see Lehto and OinasKukkonen [18] and Zwinderman et al. [36].

Another interesting field of persuasive technology research is linked to artificial intelligence e.g. making the response from persuasive system better through computational models of argument [13], or learning agents [27]. While there are several challenges associated with persuasive system design, one noted issue engaging researchers is the framing effect. Martinez and Fieulaine [22] did one interesting study on the framing effect: they explored what role the time perspective had in persuasive communication since sustainable behaviour is future-oriented. Now, one of the broadest and comprehensive goals of the persuasive system is convincing users to behave more sustainably [7] and for the sustainability problems framing is important for understanding human cognition and mind to make decisions on how to act in the future.

In regard to this, Fagley and Miller [9] found an important outcome of framing effects on user's choice. The core idea of the framing effect is that, "people's decision varies" and is not always rational and can be subject to the construction (framing) of different alternative choices [14,15,24]. Framing effects are ubiquitous in our day to day life, despite literature suggesting that, in decision making, an important role can be given to the framing effect phenomenon [20]. This makes it a challenge for persuasive system designers to understand the framing effect in an improved way. Doing this successfully would improve the persuasive design and thereby have an impact on the success ratio of a system for reaching its established sustainability goals. The fact of achieving sustainability through persuasion is criticized many times. One major reason is due to its narrow view on sustainability, focusing on "individual consumer behaviours" only; bringing challenges to sustainable HCI research, as the present persuasive research is not taking us towards any new invention, but repeating the old ones only by not producing solutions 
to them [2]. Hence, present patterns of persuasive technology have been criticized as not being adequate for sustainable system-design solutions $[2,17]$. A call for expanding the scope of persuasion towards sustainability is therefore evident, but doing this would clearly increase system complexity. New diverse types of framing effects would result to be handled due to this complexity, and a proper realization of them would then define the successfulness of the system. Nevertheless, comprehending the complex framing effect is difficult and needs thoughtfulness. This is why the need for a unifying framework that brings together different types of framing effects was addressed by Druckman [8] to be an important issue. In addition, persuasive sustainability can be seen as a problem that is associated with non-extreme probabilities that's characterizing one particular sustainability problem in multiple ways. Studies involving non-extreme probability problems showed promising effects of framing on user choice [9], although the predicted direction from prospect theory and the response of these studies were not so consistent [24]. To put it differently, framing the sustainability problems in a positive and negative scale is not enough for the success of persuasive sustainability. These were the underlying ground behind the key research question of this paper, "how to manage framing effects in designing persuasive systems for sustainability?" As an answer to this question, the authors used previous theoretical frameworks for proposing a model of framing effects, which would be useful for managing different complex framing effects effectively in persuasive design. Followed by the model, this paper also offers our recommendation for different framing effect management principles for persuasive sustainability.

This essay is structured as follows. A background in Section 2 is presented shortly discussing the framing effects and its importance in persuasive system design. The theoretical model is presented in Section 3 while Section 4 discussed the principles of framing effect management derived from the theoretical model. Section 4 also presents different sustainability dimensions and their relation to the proposed management principles and a design space for persuasive sustainability. Finally, discussions, future work directions and conclusions were given in Section 5 and Section 6 .

\section{BACKGROUND}

\subsection{Framing effects}

Tversky and Kahneman [34] used the term "decision frame" to describe a decision maker's conception of acts, results, and contingencies associated with a particular choice. Norms, personal characteristics and habits control partly the frame that a decision-maker adopts. One frame effect that has been studied is invariance. Invariance is the assumption that choice is not stimulated by variations of irrelevant features of options or outcomes [34,35]. Framing effect is thus a type of cognitive bias that is used from basic advertising in everyday life to complex biasing involved in politics. Recent advanced research by Levin et al., [20] showed framing effects to be operational in behavioural and neuroscience research by playing a major role in understanding risk-focused neuro-economic decision processes. Variations in the framing of acts, contingencies, and outcomes can reverse preference [34]. The framing of act effect states that choices involving losses are often risk taking and choices involving gains are often risk averse. It is possible to impact on the user's choice by the framing of the act in the right way. The framing of contingencies is seen in the response pattern following occurrences of choice. The framing of outcome is when, in relation to a reference neutral outcome, other outcomes are commonly perceived as either negative or positive and changing of the reference point will also change the evaluation of the outcome. The difference between an option that is framed as a disadvantage or advantage will differ because the value function is steeper for losses than for gains. Often people evaluate acts in terms of minimal accountability (i.e. they only see the direct consequence of the act) and makes selection faster because it simplifies the evaluation process and gives a reduced cognitive strain. Research about outcomes on choice varies, e.g. Tversky and Kahneman [34] accounted framing for approximately $25 \%$ of the variance in choice, while Siminoff and Fetting [32] found it accounting for less than $3 \%$, hence the framing effect in persuasive design for sustainability will vary.

\subsection{Persuasive Design and Framing Effects}

Persuasive design for sustainability, with respect to framing effects, would deal with controlling the framing effects in order to change user behaviour for acting towards the sustainability goals. For shaping the behavioural three-principle factors, namely, motivation, ability, and triggers, can take a major role, as shown by Fogg [10] in a proposed behavioural model. In the Fogg behavioural model (FBM), motivation and ability are conditions that must exist before the intended behaviour that will follow, and a "trigger" must be presented to enable the occurrence of the target behaviour. By using the framing effects ingeniously it would be possible to change user's cognition and framing in line with sustainability goals. To understand the framing of an act is vital, since otherwise the designer of the system could make users choose erroneously, and behave and act in a conflicting way to the set sustainable goals. In persuasive design for sustainability, both risk aversion and risk taking could be used as strategies to persuade users. It is often possible to frame protective action in either unconditional or conditional form by controlling the level of the outcome, and this is important for persuasive design. It is difficult to know if people will value the reduction of risk too little or the elimination of risk too much, so the designer should act cautiously when considering contingency and its effects. A designer of a persuasive sustainability system should take into consideration the framing of reference points, since it would have a large impact on how a user evaluates different alternatives, and then take actions accordingly. In a literature review by Hamari et al. [12], the question whether persuasive technology really does persuade little support for an actual change of attitude is found, but they do acknowledge that the systems seem to be able to change behaviour. One interesting point is the notion that it seems like persuasive technologies are aimed at encouraging activities that the users would try to attain regardless of the system [12]. With effective framing, the user of the system could better assimilate the behaviour as something worth attempting and, in the process, change the attitude. In persuasion for a healthier behaviour, Rothman and Salovey [30] found framing and understanding of the context as important for success, and this should also hold true in persuasive design for sustainability. Both often have less impact immediately, but instead it could be important in the future. For designers, to understand how to manage 
Table 1: Persuasive design properties with different corresponding framing effects

\begin{tabular}{|c|c|c|c|}
\hline $\begin{array}{c}\text { Persuasive Design } \\
\text { Properties }\end{array}$ & Attribute Framing Effects & Goal Framing Effects & $\begin{array}{c}\text { Risky Choice } \\
\text { Framing Effects }\end{array}$ \\
\hline Equitability & Community Image & Sense of Overview & Social Practice \\
\hline Resources Use & Reasonable Benefits & Resource Stability & Noble Contribution \\
\hline Inclusiveness & Design for All & Mainstream Practice & Mental Exclusion \\
\hline Diversify Design & Multiple Focused Groups & Prohibiting Risk & Latitude Reduction \\
\hline Optimality & Simple Customization & Inclusion in Process & Exclusion from Cause \\
\hline Mutual Capability & Joint Growth & Community Move & Group Incompetence \\
\hline Privacy & Contextual Uncertainty & Protective Design & Trust Minimization \\
\hline Risk & Risk Presentation & Minimized Hazard & Improved Confidence \\
\hline Transparency & Controlling Functions & Feedback Process & Mutual Openness \\
\hline Multiple Outcomes & Transparent Communication & Justify Target,Cause & Strategy Identification \\
\hline
\end{tabular}

different framing effects would be central in this case, and this process would be easier to understand and practice with the help of a framing effect model, which is formulated and discussed in the next section.

\section{A MODEL OF FRAMING EFFECT}

\subsection{Identification of Different Factors}

For the formation of our theoretical framework, two previous studies were selected as the basis. In a study of Mustaquim and Nyström [26], design principles for persuasive design for sustainability were proposed, these principles where derived from the persuasive system design (PSD) Model [29], design strategies to promote public interest in collectivist society [16], and sustainable system design principles [25]. Levin et al. [21] identified three types of framing effects and discussed in the form of a typology. We have analysed the existing design principles of persuasive design from Mustaquim and Nyström [26] to choose key properties from them. We then, for each of the selected properties, have assigned keywords of possible consequences in the context for three different framing effect types from Levin et al. [21]. The three framing types are as follows: "Attribute" gives an event/object attribute that can be evaluated and compared, "goal" is affected by the consequence of behaviour and its impact, and "risky choice" sets different options based on the risk preference [21]. Table 1 presents the identified characteristics of the persuasive design principles for sustainability and their corresponding three framing effects. These properties are then discussed in detail.

Equitability: To be a part of a larger community is important for users in getting their attitudes biased towards sustainability, or as Cialdini [4] wrote, "people follow the lead of similar others". Of course, social pressure and normalization can inflict their attitudes that can be used in the attribute framing. In other words, the community image is an attribute framing effect, which can play positive roles in user's behaviour shaping. It is important for users to feel that by using a persuasive system they will be part of a bigger community that is working on an important cause (sustainability), but individual action must simultaneously be acknowledged as important too. A sense of generalization is important to be framed as a goal. If presented positively, this goal should bias the user towards the cause, while a negative presentation would result in negative consequences, such as being inactive in using the system. Likewise, for the user, it is important to realize that they are in a social practice by working towards sustainability. By framing the social practice phenomenon as a risky choice, it will be possible to make the users understand that if they don't act on the cause, they would be dropping their community images, and this would trigger them towards framed sustainability acts.

Resources Use: Distribution of different resources and its proof in persuasive design has been crucial for shaping user behaviour, because from the community image, the user wants to see individual and mutual benefits. Cooperation would then be a better strategy than introducing competition in the framing. How equally different resources are used can be framed positively and failure to do it could negatively influence the user in shaping their behaviour. A realization of working for stabilizing resource uses is an important goal of sustainability, and persuasive system should act on that framing effect. Acting on sustainability is a noble cause, and this can be framed as a risky choice framing effect. If users do not act on this then they will miss doing something noble, which triggers their act towards the sustainability cause.

Inclusiveness: Framing the target user groups within the context of including the most possible users, regardless of their physical or psychological abilities, add value into the design. Individuals then feel motivated to act towards the cause by being the part of a bigger community, not being alone and not having any impacts. Practising design for all has an important reasoning towards main-streaming universal design and this can be a central goal for user motivation. In the same way, by not being included in the process of universal design, users can be psychologically excluded from a cause, which can be framed within the context of risky choice.

Diversify Design: Diversification of design by multipleuser group consideration increases the inclusiveness of an individual, and therefore, positive framing of this is important. On the other hand, different risks can be increased by not being included in the diversifying domain. This is an important goal framing effect for users in design diversification. Risky choice can be framed by presenting that users could be in the heightened risk of narrowing down their latitude with others in the community and thus motivate them acting on the sustainability cause.

Optimality: Miscellaneous users have unalike capabilities, and allowing users the ability to customize a system plays a big role in persuasion acts. This ability can be a positive framing effect. Goal framing effect can be presented as being included in a bigger process in the community for the 
optimal use of a system towards the specified cause while a risk effect could be framed by arguing that users could be excluded from the process of achieving sustainability.

Mutual Capability: Including users in a design process are mostly beneficial, but users do not want to feel neglected in this process. A linking growth in the community, other users and design team can initiate the realization of mutual capabilities to the users. Framing this factor positively can play a role in successful persuasive design for sustainability. Here, the goal is acting towards moving a community for the cause of sustainability. Risk framing effect could be failure to act towards this goal and would make a user feel incompetent to be able to work in the group.

Privacy: While privacy is important in persuading, different issues regarding privacy could be simply contextual and user may not be comfortable about them. Uncertainties regarding privacy under different contexts are therefore important to frame as an attribute for increasing confidence and reliability of users on the persuasive system they would use. While there are always some privacy issues associated with system design, users need to be clearly informed that the designed system is well protected for maintaining privacy and this can be framed as the goal. If users still feel insecure, doubtful and less confident about the system, then, the issues associated with trust minimization can be framed as the risk framing effect, clarifying that a user might drop trust credibility within a community. Thus, losing trust can work as a risky choice factor towards user behaviour shaping for persuasive sustainability.

Risk: Privacy is not the only risk that users can think of while choosing a persuasive system, but there could be other associated issues, namely, the after effect on behaviour, adverse effect due to the use of a system, etc. Presenting the possible associated risks in a frame is thus important to boost the user's confidence towards the use of a system. Users need to be informed that the hazard of using a specific system is minimized by showing comparison with other similar systems, which can be a goal framing effect. On the other hand, by not taking smaller risks, users could be presented with the fact that they are struggling with their confidence, comparing with other associated users in the same community, resulting in this being a risky choice framing effect in this case.

Transparency: Designed persuasive system needs to be transparent, yet at the same time, it needs to inform users how different functions are being handled. Function controlling should be framed and presented clearly for increasing the system transparency. A goal that can work as a trigger towards user activeness could be showing the importance of individual abilities in a feedback system for improving a system design, and thereby result in sustainability. A negative risky choice could be clarifying the importance of being mutually open to other users and thereby triggering them to act towards transparency. This act can contribute in finding different feedbacks, identifying synergies and can be used for existing persuasive system improvement.

Multiple Outcomes: While a persuasive system can act on user's behaviour towards sustainability, it might not be the only outcome of the system and other associated outcomes could also appear. Transparency in communication should therefore be framed to make users become clearly aware of different consequences. This would stop the users from acting against using the system, if at some point sit- uation arises which they were not prepared to face. It is also important here to frame the cause (sustainability) for users in a justified manner which can work as a goal framing effect. It can then be shown that a failure to be included in the process of the different strategic identification could be a negative and risky choice. This will trigger users to act on transparent communication with other users for sharing critical information, resulting in the improvement in a persuasive system and acting towards sustainability.

Based on the above discussions of the properties of three framing effects for different persuasive design principle characteristics, a model can be structured as shown in Figure 1 and described in section 3.2.

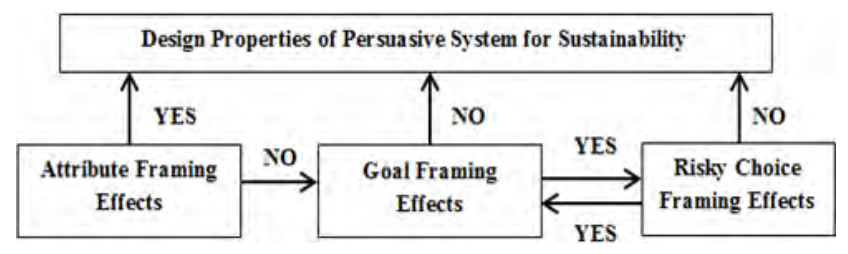

Figure 1: A framing effect management model for persuasive design.

\subsection{Proposed Model}

From Table 1 it was understood that different properties of persuasive design could be re-elected to different types of framing effects and the proposed model in Figure 1 shows them in the form of an abstract theoretical model. The fundamental principle here is the concept of building or improving the knowledge of persuasive design principles by using properties of different framing effects in an iterative process. This would deepen the analysis of the persuasion context and benefit the selection of persuasive design principles, which is the first generic step in persuasive system development [29]. The "yes" and "no" notions were used to represent positive and negative framings. Since attribute framing effects are about favouring positive framed attributes, it is shown directly connected with the object of adding value into the different persuasive design principles for sustainability. A negative framing for attribute has no direct relation to improved understanding of the design principles, and therefore should follow towards goal framing effect. At this time for goal framing effect, the persuasion is in focus and is about reflecting positive results of a performed act with the negative consequences of not performing an act. The latter is important in persuasive sustainability and is shown directly connected with the design principles. In addition, positive consequence follows the risky choice framing effect where the aim is to persuade the users (to take risks) by showing a negatively framed message, which is why it is shown to be connected with the design principles. However, a positive framed message can increase failure in the case of risky choice framing effect since the associated risks would not be presented properly and would lack proper persuasion. Smith and Petty [33] found support in their research and also observed in previous research that potential losses (negative framing) in some context worked better for persuasion than potential gains (positive framing) (e.g. [21,23]) although the opposite was observed in another context (e.g. [6,31]). Thus, it is important to understand the context of the persuasive 
system. This is why this consequence is shown as a return to the goal framing effects. In an iteration, goal could then be framed in an improved way for the success of persuasiveness. Of course the process can iterate if needed for achieving required success in persuasion.

In contrast, it is important to explain why a negative framing of attributes or a positive framing for goal is connected to the subsequent framing effect types. In persuasive sustainability, attribute framing is usually a very straight forward approach (yes or no) and users might not be convinced in the beginning through the representation of a message in this way. A failure would therefore lead towards the goal framing effect where the framing is done in a goal oriented way. Here the users are more likely to get biased towards the sustainability causes, since the presentation would focus on the consequences of negativity as a trigger. At the same time, if the goal framing effects fail to impact on users, the option moves into the risky choice framing effect. Here, different associated risks would be presented to the users as a challenge that they would accept as a trigger towards sustainability. Similarly, a failure with risky choice framing would move to the goal framing, where the process clarified earlier would repeat. The framing would also depend on the information given by the system e.g. Das et al. [5] found different effectiveness for fundraising based on if the information was anecdotal (best combined with positive framing) or abstract (best with negative framing).

The process in the model can thus be summarized by stating the fact that the identification and selection of different design principles and realization of their associated properties of the persuasive sustainability design could be supported through a proper management of different types of accompanying selected framing effects with the help of this proposed model. If artificial intelligence is used in the designed system, it should be possible for the system to adapt to and learn from the user's interaction with the system, similar to Narita and Kitamura's [27] learning agents or Hunter's [13] argument-centric persuasion, in order to enhance the framing and increase the success rate of the persuasion. Moreover, the illustration of the model is very abstract and theoretical which makes bringing it into practice more challenging. Some principles of framing effect management are therefore needed. The following section proposes principles of framing effect management that is summarized based on the contents from Table 1 and the structure of the model in Figure 1. Since the success of a persuasive system can be highly dependent on the way it is used [29], the way it will be designed would eventually impact on deciding the characteristics of the end system. Doing this can thus define the system's success in use. The following management principles should therefore be perceived as reflecting their importance in situations involving the design of persuasive design for sustainability only.

\section{FRAMING EFFECT MANAGEMENT PRINCIPLES}

In this section, the framing effect management principles that were formulated from the proposed model are discussed in detail, then a relationship of different sustainability dimensions with the properties of the principles are shown, and finally, a design space for persuasive sustainability are presented.
Mainstream social practice: The image of a community must be balanced and make sense to the community members that are users of a persuasive system. If the community image is perceived to be too strong, people could feel excluded or make other people leave from activities. Both the community and individuals must be stressed as important for reaching the sustainability goal to persuade in taking action towards a sustainable goal.

Heuristic representation of resources: Since the efforts needed for using a system needs resources, the benefits for both the individual and mutual should be viewed as reasonable, and there is no need to exaggerate. People want to be seen as good and that they do things for the right cause. This perception of doing something noble could be used as an effective trigger.

Legitimation of design for all: By framing the system as inclusive and designed for all the legitimacy increases, since it is not seen as something that only a few initiated or elite should get involved with. Neither by doing this it narrows down the scope for people with special needs or specific community only, but the opposite would happen. If a system is not seen to be an inclusive one, then there is a risk that possible future important users would choose not to get engaged.

Group inclusion importance: If multiple focus groups are included, the risk of surprises decrease since the probability of missing some important changes in the use or context of the system will be low compared to only observe specific groups. Representation of this importance can be framed with a different way for users and can reveal important design properties that can be used to design a persuasive system.

Degree and ease of use: Users have different cognitive preferences for interacting with the system. A flexible system assures configuration and customization for a majority of users to fit for them. If the system lacks the possibility of customization, some groups of users might feel excluded and be lost to the sustainability cause.

Collaborative growth practice: By including users from the beginning of a process in design will make them more committed to the cause since they will internalize the goal and feel that they took an active part in the development of the system towards the addressed cause. Thus, the users will grow alongside the system and be more active in the future development.

Confidence in privacy: It is important to be straightforward and open regarding the privacy issues. The user must easily understand the privacy policies and how information gathered from the system can and will be used. If users don't trust the system or if the policies about privacy are changed they might feel tricked. In the contrary, some users will avoid the system and others who use it will choose to leave.

Transparent representation of risk: The transparency of the system needs to be high for the reduction of user's uncertainty about results of different actions. Fast and accurate feedback from the system should be stressed as a remedy for this issue. Associated risks if there are any, should be tackled and the level of risks should be rationally presented to persuade users for thinking positively towards using the system.

Communication openness: The system could impact many things: not only the sustainability goal. It's important 


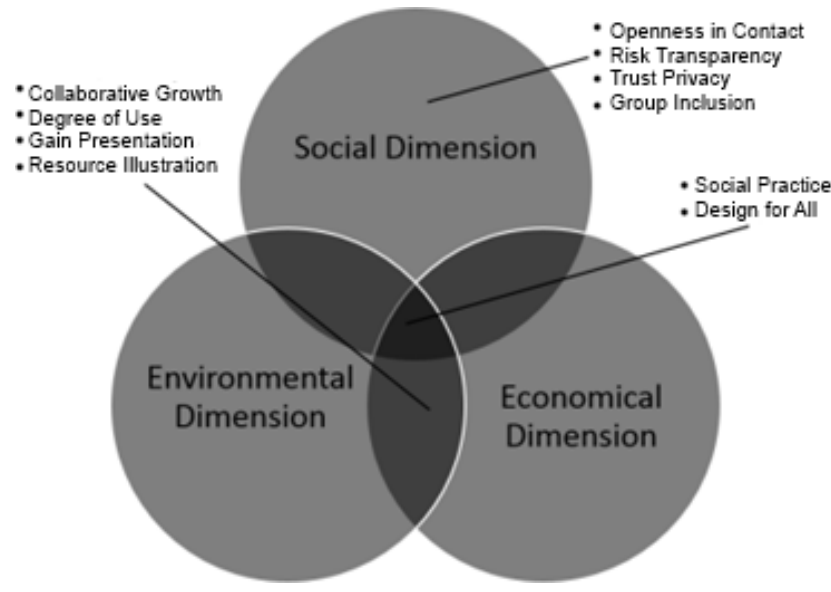

Figure 2: Sustainability dimensions and framing effect management principles.

that users get a full picture and understand any side effects that could happen for being prepared. By getting a holistic understanding, the user can justify the sustainability goals and be persuaded towards selecting their best strategies to take part in the complex and continuously changing world.

General advantage demonstration: The users will be more interested to participate if it is possible to understand and see the kind of effect that a commitment to the persuasive system could have on sustainability. If successful, the persuasive system will create active and loyal users that will give support to the system and help in the future development.

Figure 2 shows the three dimensions of sustainability where corresponding key properties from the above described principles are shown. In Figure 3, each key property from the framing effect management principles were shown in the form of a persuasive sustainability design space. Figure 2 clarifies how social, environmental, and economic pillar of sustainability can be mapped together with the properties of our identified framing effect management principles. While four factors can be seen to be directly associated with a social dimension, four other factors have commonality and influence in economic and environmental dimensions, while two factors are shown to have influence on all three dimensions of sustainability. While there are numerous identifiers proposed in present literature to quantify sustainability, these ten identified factors originated from the relationships of design principles, and three different types of framing effects could be used as a set of specific identifiers for evaluating sustainability achieved through the use of persuasive design. Similarly, the efficiency of a persuasive design for sustainability could be evaluated by measuring these factors. The design space shown in Figure 3 can shed some lights on using the identified factors in persuasive design for sustainability and its evaluation.

Figure 3 illustrates a conceptual design space that shows the possible available design options for persuasive sustainability. The proposed design space is based on the ten identified principles of framing effect management. By using reverse engineering, it is also possible to improve the understanding of each framing effect management principles

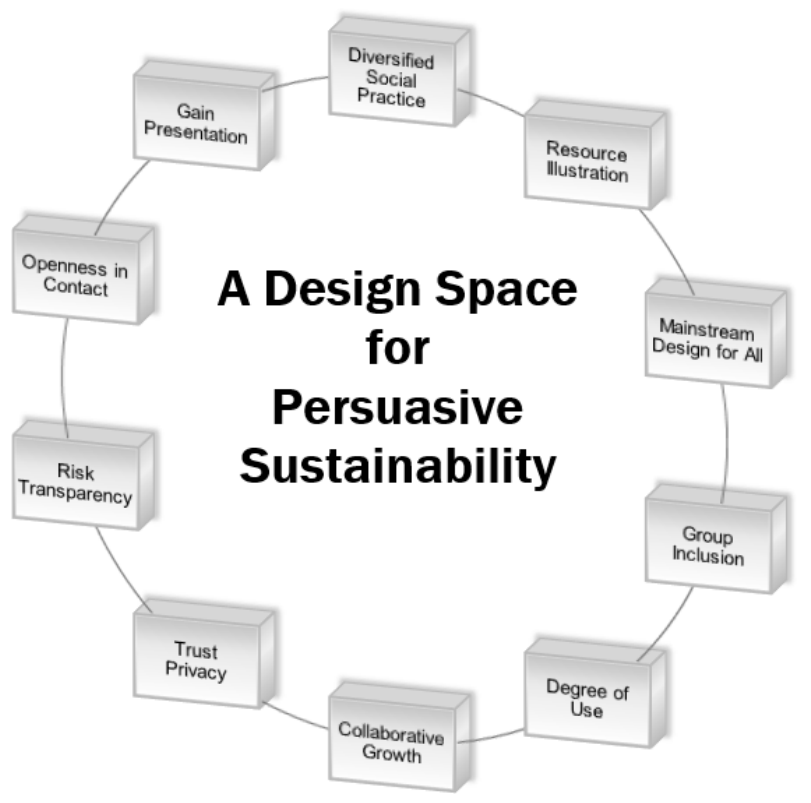

Figure 3: A design space for persuasive sustainability.

for improving the perception of the process in our proposed model. It is important as a thorough analysis and understanding of the principles can enhance design and then build a successful persuasive system for sustainability.

\section{DISCUSSIONS}

Managing framing effect is a complex mechanism where predicting the right outcome is difficult. In a certain context one framing will be successful but the same approach will not achieve persuasion, just like previous research have shown $[6,19,23,30,31]$. It seems evident that in persuasion, the framing effect plays a major role and understanding them is crucial for improved design. Unintended outcomes can provide learning opportunities for designers. This learned knowledge can be used in iteration of a design process and further designing similar systems. In persuasion, the framing effect and its proper use can play a positive role in the result towards a cause like sustainability. Obviously, avoiding different biases that are not good is important, but another way of looking framing effects is, to take the most out of different biases (managing them) for understanding users and generate new design knowledge in the form of improved design principles. This concept was used for the analysis of different associated framing effects in this paper. The analysis showed in Table 1 is complex. Our interpretation of the outcomes of different framing effects on each design principle characteristic could be interpreted in different ways. We, however, took a heuristic approach and identified the factors after critical analysis of different consequences in persuasive sustainability. The proposed model, then shed light on the issues of treating different types of framing effects in the form of a theoretical process. The management principles for framing effects that were proposed later is the outcomes from this model. Levin et al. [21] argued the importance of considering attribute framing together with the goal or risky 
choice framing for an improved persuasive communication. Our model supports this argument, since we showed how different framing effects could be connected in an iterative process from where new knowledge identification for persuasive design principles could be benefited. Again, Cassotti et al., [3] found that in the decision making, it is possible to eliminate different framing effects if the emotional context is positive. Our identified different characteristics from the proposed model discussed earlier supports this argument. That is to say, by using the knowledge of managing different framing effects, a positive emotional experience would be created for the users (through the persuasive design) which would in turn act towards eliminating other negative framing effects and thereby act towards making decisions for the cause of sustainability.

On the other hand, the relationship of the different sustainability dimensions and principles of attribute management is a unique contribution presented in this paper. This relationship can be interpreted from a few different perspectives. Firstly, it would be possible to select a set of characteristics based on the dimensions of sustainability for conducting design. Therefore, it can be argued that selected characteristics would result in a specific dimension focused sustainability through the end design. Secondly, as stated earlier, the addressed characteristics could be seen from the perspective of a sustainability identifier, and a specific dimension focused, persuasive design could thus be evaluated by using the corresponding characteristics. Finally, for an existing persuasive design for sustainability, different dimensions could be evaluated to see which dimensions of sustainability need improvement to support any gaps in sustainability achievement.

Biskjaer et al. [1] argued for using the designer's own experience and knowledge in response to different external conditions to construct a design space. Our formulated design space in Figure 3 could be supported by this argument, since identified characteristics of different management principles were used to formulate the design space, where external conditions were critically considered in the context of framing effect resulting the design space to be a guidance for the designers. On the contrary, the purpose of this paper is to contribute theoretically and reflect on an important issue that has not been addressed previously, without bringing any empirical verification. Evaluation of the design principles is thus the next step and would be the main goal of future research. Evaluation can also show the relevance of the different principles, that is, some principles could be more important than others and analysis could reveal connections between different principles. Another challenge is to analyse whether these principles could be generalized in persuasive design for sustainability or not. Another possibility is that proposed principles would improve a persuasive sustainability system design when focusing a closed user group, while resulting in no or partial influence on another user group. Finally, advance studies could always be performed with brain imaging or eye tracking where newly developed complex, risky choice framing effects like neuro-economic tasks [20] could be considered in an economy focused persuasive sustainability.

\section{CONCLUSIONS}

Persuasion for sustainability is important, but has its own critics. If we want to expand and improve the scope and de- sign of persuasive sustainability, complex issues like framing effects needs to be considered. In this paper, we have formulated a framing effect management model for persuasive sustainability design. We have used two previous theoretical frameworks from where different characteristics of persuasive sustainability design principles were identified. We then have analysed them in the context of three different types of framing effects (attribute, goal and risky choice). The process associated with the proposed model resulted design principles for managing framing effects in system design for persuasive sustainability also proposed and discussed in the paper. We argued that using the developed principles for effective framing management it should be possible to raise the success ratio of a persuasive system. We then have shown the logical relationships of different properties from these principles with different dimensions of sustainability leading towards the formation of a design space for persuasive sustainability. We believe that the use of the proposed principles and successful mapping of the model would considerably improve the probability of reaching a set sustainability goals through persuasive design. Extensive experiments are therefore needed for verifying the different associated impacts of the identified factors from our proposed theoretical model.

\section{REFERENCES}

[1] Biskjaer, M. M., Dalsgaard, P., and Halskov, K. A constraint-based understanding of design spaces. In Proceedings of the 2014 Conference on Designing Interactive Systems (DIS '14), ACM, New York, 2014, 453-462.

[2] Brynjarsdottir, H., Håkansson, M., Pierce, J., Baumer, E., DiSalvo, C., and Sengers, P. Sustainably unpersuaded: How persuasion narrows our vision of sustainability. In Proceedings of the SIGCHI Conference on Human Factors in Computing Systems (CHI '12), ACM, New York, 2012, 947-956.

[3] Cassotti, M., Habib, M., Poirel, N., Aïte, A., Houdé, O., and Moutier, S. Positive emotional context eliminates the framing effect in decision-making. Emotion, 12(5), 2012, 926-931.

[4] Cialdini, R. B. Harnessing the science of persuasion. Harvard Business Review, 79(9), 2001, 72-81.

[5] Das, E., Kerkhof, P., and Kuiper, J. Improving the effectiveness of fundraising messages: The impact of charity goal attainment, message framing, and evidence on persuasion. Journal of Applied Communication Research, 36(2), 2008, 161-175.

[6] Davis, J. J. The effects of message framing on response to environmental communications. Journalism \& Mass Communication Quarterly, 72(2), 1995, 285-299.

[7] DiSalvo, C., Sengers, P., and Brynjarsdóttir, H. Mapping the landscape of sustainable hci. In Proceedings of the SIGCHI Conference on Human Factors in Computing Systems (CHI '10), ACM, New York, 2010, 1975-1984.

[8] Druckman, J. N. The implications of framing effects for citizen competence. Political Behavior, 23(3), 2001, $225-256$

[9] Fagley, N. S., and Miller, P. M. The effect of framing on choice interactions with risk-taking propensity, cognitive style, and sex. Personality and Social 
Psychology Bulletin, 16(3), 1990, 496-510.

[10] Fogg, B. J. A behavior model for persuasive design. In Proceedings of the 4th International Conference on Persuasive Technology (Persuasive '09), ACM, New York, 2009, 40:1-40:7.

[11] Fogg, B. J., Cuellar, G., and Danielson, D. Motivating, influencing, and persuading users: An introduction to captology. In Human Computer Interaction Fundamentals, A. Sears and J. A. Jacko, Eds. 2009, 109-122.

[12] Hamari, J., Koivisto, J., and Pakkanen, T. Do persuasive technologies persuade? - a review of empirical studies. In Persuasive Technology, A. Spagnolli, L. Chittaro, and L. Gamberini, Eds., vol. 8462 of $L N C S$. Springer International Publishing, $2014,118-136$.

[13] Hunter, A. Opportunities for argument-centric persuasion in behaviour change. In Logics in Artificial Intelligence, E. Fermé and J. Leite, Eds., vol. 8761 of LNCS. Springer International Publishing, 2014, 48-61.

[14] Kahneman, D. A perspective on judgment and choice: mapping bounded rationality. American psychologist, 58(9), 2003, 697-720.

[15] Kim, S., Goldstein, D., Hasher, L., and Zacks, R. T. Framing effects in younger and older adults. The Journals of Gerontology Series B: Psychological Sciences and Social Sciences, 60(4), 2005, P215-P218.

[16] Kimura, H., and Nakajima, T. Designing persuasive applications to motivate sustainable behavior in collectivist cultures. PsychNology Journal, 9(1), 2011, 7-28.

[17] Knowles, B., Blair, L., Walker, S., Coulton, P., Thomas, L., and Mullagh, L. Patterns of persuasion for sustainability. In Proceedings of the 2014 Conference on Designing Interactive Systems (DIS '14), ACM, New York, 2014, 1035-1044.

[18] Lehto, T., and Oinas-Kukkonen, H. Persuasive features in six weight loss websites: A qualitative evaluation. In Persuasive Technology, T. Ploug, P. Hasle, and H. Oinas-Kukkonen, Eds., vol. 6137 of LNCS. Springer, 2010, 162-173.

[19] Levin, I. P., and Gaeth, G. J. How consumers are affected by the framing of attribute information before and after consuming the product. Journal of consumer research, 15(3), 1988, 374-378.

[20] Levin, I. P., McElroy, T., Gaeth, G. J., Hedgcock, W., and Denburg, N. L. Behavioral and neuroscience methods for studying neuroeconomic processes: What we can learn from framing effects. In The Neuroscience of Risky Decision Making, V. F. Reyna and V. Zayes, Eds. American Psychological Association, 2014, 43-69.

[21] Levin, I. P., Schneider, S. L., and Gaeth, G. J. All frames are not created equal: A typology and critical analysis of framing effects. Organizational behavior and human decision processes, 76(2), 1998, 149-188.

[22] Martinez, F., and Fieulaine, N. Time and the misfits: Temporal framing and priming in persuasive communication. In Time Perspective Theory; Review, Research and Application, M. Stolarski, N. Fieulaine, and W. van Beek, Eds. Springer International
Publishing, 2015, 385-402.

[23] Meyerowitz, B. E., and Chaiken, S. The effect of message framing on breast self-examination attitudes, intentions, and behavior. Journal of personality and social psychology, 52(3), 1987, 500-510.

[24] Miller, P. M., and Fagley, N. S. The effects of framing, problem variations, and providing rationale on choice. Personality and Social Psychology Bulletin, 17(5), 1991, 517-522.

[25] Mustaquim, M., and Nyström, T. Designing sustainable it system -from the perspective of universal design principles. In Universal Access in Human-Computer Interaction. Design Methods, Tools, and Interaction Techniques for eInclusion, C. Stephanidis and M. Antona, Eds., vol. 8009 of LNCS. Springer, 2013, 77-86.

[26] Mustaquim, M., and Nyström, T. Designing persuasive systems for sustainability - a cognitive dissonance model. In Proceedings of the European Conference on Information Systems (ECIS) 2014, AISeL, 2014.

[27] Narita, T., and Kitamura, Y. Persuasive conversational agent with persuasion tactics. In Persuasive Technology, T. Ploug, P. Hasle, and H. Oinas-Kukkonen, Eds., vol. 6137 of LNCS. Springer, 2010, 15-26.

[28] Oinas-Kukkonen, H., and Harjumaa, M. Towards deeper understanding of persuasion in software and information systems. In International Conference onAdvances in Computer-Human Interaction 2008 , IEEE, 2008, 200-205.

[29] Oinas-Kukkonen, H., and Harjumaa, M. Persuasive systems design: Key issues, process model, and system features. Communications of the Association for Information Systems, 24(1), 2009, 485-500.

[30] Rothman, A. J., and Salovey, P. Shaping perceptions to motivate healthy behavior: the role of message framing. Psychological bulletin, 121(1), 1997, 3-19.

[31] Rothman, A. J., Salovey, P., Antone, C., Keough, K., and Martin, C. D. The influence of message framing on intentions to perform health behaviors. Journal of Experimental Social Psychology, 29(5), 1993, 408-433.

[32] Siminoff, L. A., and Fetting, J. H. Effects of Outcome framing on treatment decisions in the real world: Impact of framing on adjuvant breast cancer decisions. Medical Decision Making, 9(4), 1989, 262-271.

[33] Smith, S. M., and Petty, R. E. Message framing and persuasion: A message processing analysis. Personality and Social Psychology Bulletin, 22(3), 1996, 257-268.

[34] Tversky, A., and Kahneman, D. The framing of decisions and the psychology of choice. Science, 211(4481), 1981, 453-458.

[35] Tversky, A., and Kahneman, D. Rational choice and the framing of decisions. Journal of business, 59(4), 1986, S251-S278.

[36] Zwinderman, M. J., Shirzad, A., Ma, X., Bajracharya, P., Sandberg, H., and Kaptein, M. C. Phone row: A smartphone game designed to persuade people to engage in moderate-intensity physical activity. In Persuasive Technology, M. Bang and E. L.

Ragnemalm, Eds., vol. 7284 of LNCS. Springer, 2012, $55-66$. 